\title{
Driving glioblastoma growth by alternative polyadenylation
}

\author{
Cell Research (2014) 24:1023-1024. doi:10.1038/cr.2014.88; published online 4 July 2014
}

Global shortening of $\mathbf{3}^{\prime}$ untranslated regions ( $3^{\prime}$ UTRs) through alternative polyadenylation is an emerging hallmark of cancer. A recent study identifies the cleavage factor Im 25 (CFIm25) as an important mediator of 3' UTR shortening in glioblastomas and demonstrates a causal relationship between alternative polyadenylation and cancer cell proliferation.

Virtually all messenger RNAs (mRNAs) in eukaryotes are cleaved and polyadenylated at their $3^{\prime}$ ends [1]. The site of polyadenylation defines the length of the $3^{\prime}$ untranslated region ( $3^{\prime}$ UTR), which contains numerous regulatory elements that are recognized by RNA-binding proteins and small noncoding RNAs to influence the stability, localization, and translation of mRNAs. Alternative cleavage and polyadenylation of mRNAs can generate different 3' UTR isoforms as a means to engage or evade 3' UTR-mediated regulatory controls. Recent genome-wide studies in multiple eukaryotic species, ranging from yeast to humans, have revealed the prevalence of alternative polyadenylation, with $>50 \%$ of mRNAs possessing more than one $3^{\prime}$ UTR isoform [2, 3]. Understanding the roles of pervasive alternative polyadenylation and 3' UTR isoform usage in physiological and pathological conditions, as well as the mechanisms regulating alternative polyadenylation, has become an area of intense study.

Global changes in $3^{\prime}$ UTR lengths have been observed in three major biological systems. First, rapidly proliferating cells are found to express mRNAs with shorter 3' UTRs. For example, during $\mathrm{T}$ cell activation, there is an increase in the expression of $\mathrm{mRNAs}$ terminating at upstream polyadenylation sites, suggesting that alternative polyadenylation may coordinate gene expression with rapid cellular proliferation [4]. Second, during animal development, many genes switch their 3' UTR isoform usage at developmental transitions, indicating that different $3^{\prime}$ UTR isoforms may confer stage-specific regulation to their host genes [2]. Third, among different organs in animals, the brain tends to express the longest 3' UTR isoforms [5]. For example, many 3' UTR isoforms expressed in the mammalian brain can extend to $>10$ kilobases in length. The biological function and regulation of these longer neuronal 3' UTRs is not well understood. In addition to these physiological processes, global alterations in $3^{\prime}$ UTR lengths have been observed in many types of cancer. In comparison with non-transformed cells, cancer cells often express mRNA isoforms with shorter 3' UTRs. Some of these mRNAs encode oncogenes, whose expression is often elevated as a result of 3' UTR shortening and consequent loss of negative regulatory elements, such as microRNA binding sites [6]. Despite a strong association between 3' UTR shortening and oncogenesis, it remains unclear whether global shortening of 3' UTRs is a mere consequence or a cause of tumorigenesis. In a recent paper published in Nature, Masamha et al. [7] provide compelling evidence in support of a direct oncogenic role for global 3' UTR shortening in promoting cellular proliferation and tumorigenesis in glioblastomas.

Masamha et al. first established a quantitative RT-PCR assay to evalu- ate the $3^{\prime}$ UTR lengths of three genes (CCND1, DICER1 and TIMP2) known to undergo alternative polyadenylation. They found that these three genes expressed long 3' UTRs in HeLa cells despite their highly transformed state. Using these three genes as reporters, they performed a targeted RNA interference (RNAi) screen of known factors involved in mRNA cleavage and polyadenylation. They found that RNAi-mediated depletion of members of the cleavage factor Im (CFIm) complex, in particular its CFIm25 subunit, resulted in shortening of the $3^{\prime}$ UTRs of these three genes. This finding is in agreement with a previous report showing that knockdown of CFIm68, the other subunit of the CFIm complex, systemically shortens $3^{\prime}$ UTRs [5]. To identify 3' UTRs whose lengths are regulated by CFIm25, by combining high-depth RNA-sequencing with refined bioinformatics analysis, the authors showed that, in CFIm25-knockdown cells, 1450 transcripts have shorter 3' UTRs, whereas only 3 transcripts have longer $3^{\prime}$ UTRs. They further demonstrated that the shortening of $3^{\prime}$ UTRs led to increased expression of several target genes, including several oncogenes. To validate their findings from cultured cancer cells, they interrogated RNA-seq data collected from patient-derived glioblastoma samples in the Cancer Genome Atlas (TCGA). By stratifying samples based on CFIm 25 expression levels, they observed a positive correlation between CFIm 25 expression and 3' UTR length alterations. To test whether altering CFIm 25 expression can modulate the oncogenic properties of glioblastoma cells, the authors screened a panel of glioblastoma 
cell lines and identified cell lines that express low or high levels CFIm 25 . Artificial elevation of CFIm 25 in a glioblastoma cell line expressing low levels of endogenous CFIm25 impaired anchorage-dependent growth and cellular invasion and reduced tumor growth in a mouse xenograft model. Conversely, reduction of CFIm 25 expression in a glioblastoma cell line expressing high levels of CFIm 25 enhanced cellular proliferation and tumor growth. Based on these in vitro and in vivo results, the authors conclude that CFIm25 acts as a tumor suppressor through lengthening of 3' UTRs of growth-promoting mRNAs.

CFIm25 is among a handful of recently discovered factors that regulate alternative polyadenylation and 3' UTR length. These factors can be classified into three major classes. The first class includes factors involved in mRNA 3' end processing. Their overexpression in general leads to preferential use of downstream polyadenylation sites, resulting in broad lengthening of $3^{\prime}$ UTRs [8]. The second class includes factors that affect other aspects of transcription, such as transcription elongation and splicing. One such example is U1 small nuclear ribonucleoprotein (snRNP), which is normally involved in splicing but also suppresses the use of upstream polyadenylation sites [9]. The third class includes specific RNA-binding proteins, such as the neuronal RNA-binding protein ELAV, which mediates 3' UTR extension in the Drosophila nervous system [10]. Despite these exciting discoveries, a complete mechanistic understanding of alternative polyadenylation is still lacking. Future studies employing unbiased screens in multiple biological systems, where 3' UTR lengths are dynamically regulated, will likely reveal additional factors beyond these current three classes of factors.

The fact that CFIm25-dependent 3' UTR length control has such a critical role in glioblastoma tumorigenesis makes one wonder how prevalent this mechanism is in other cancer types. Extensive mining of the vast cancer genomics datasets should provide an answer to this question and, more broadly, could potentially reveal additional features (somatic mutations, copy number variants, gene expression, etc.) that strongly correlate with $3^{\prime}$ UTR shortening in cancer. Finally, will the lengthening of 3' UTRs be a viable therapeutic strategy to treat cancer? It is conceivable that small molecules that target regulators of alternative polyadenylation to enhance the selection of downstream polyadenylation sites will likely thwart tumor growth, without hurting normal cells.

\section{Ting Han ${ }^{1}$ John K Kim²}

${ }^{1}$ Department of Biochemistry, UT Southwestern Medical Center, Dallas, TX 75390-9152, USA;

${ }^{2}$ Life Sciences Institute, Department of Human Genetics, University of Michigan, Ann Arbor, MI 48109-2216, USA

Correspondence: John K Kim

E-mail: jnkim@umich.edu

\section{References}

1 Di Giammartino DC, Nishida K, Manley JL. Mol Cell 2011; 43:853-866.

2 Mangone M, Manoharan AP, Thierry-Mieg D, et al. Science 2010; 329:432-435.

3 Ozsolak F, Kapranov P, Foissac S, et al. Cell 2010; 143:1018-1029.

4 Sandberg R, Neilson JR, Sarma A, et al. Science 2008; 320:1643-1647.

5 Miura P, Shenker S, Andreu-Agullo C, et al. Genome Res 2013; 23:812-825.

6 Mayr C, Bartel DP. Cell 2009; 138:673-684.

7 Masamha CP, Xia Z, Yang J, et al. Nature 2014; 509:412-416.

8 Martin G, Gruber AR, Keller W, et al. Cell Rep 2012; 1:753-763.

9 Berg MG, Singh LN, Younis I, et al. Cell 2012; 150:53-64.

10 Hilgers V, Lemke SB, Levine M. Genes Dev 2012; 26:2259-2264. 